\title{
Re-evaluating the conventional wisdom about binding assays
}

\author{
Brandon D. Wilson ${ }^{1}$, H. Tom Soh ${ }^{2,3,4^{*}}$
}

\begin{abstract}
Affiliations:
${ }^{1}$ Department of Chemical Engineering, Stanford University, Stanford, CA 94305, USA.

${ }^{2}$ Department of Electrical Engineering, Stanford University, Stanford, CA 94305, USA.

${ }^{3}$ Department of Radiology, Stanford University, Stanford, CA 94305, USA.

${ }^{4}$ Chan Zuckerberg Biohub, San Francisco, CA 94158, USA.

* Correspondence to tsoh@stanford.edu
\end{abstract}

Keywords:

- Binding assays

- Affinity reagent

- Digital detection

- Langmuir isotherm

- Specificity

\section{GLOSSARY:}

Affinity Reagent: A molecule that binds to a specific target molecule of interest for the purpose of detection or quantification. An affinity reagent can be an antibody, peptide, nucleic acid, or small molecule.

Aptamer: A specific type of affinity reagent that is made of DNA or RNA. They are produced through repeated rounds of directed evolution.

Binding Assay: A method of molecular quantification that involves the binding of a one molecule to another, i.e. receptor to ligand, antibody to antigen, etc. The addition of target molecule shifts the equilibrium towards a higher fraction of target-bound molecules.

Binding Curve: A response function that characterizes the amount of binding as a function of target concentration.

Detection Range: The range between the lowest to highest concentrations over which the assay output signal has a large derivative with respect to target concentration. This is often considered to be the concentrations over which the binding curve corresponds to between $10 \%$ to $90 \%$ of that total signal.

Dissociation Constant $\left(\mathbf{K}_{\mathbf{D}}\right)$ : An equilibrium constant that characterizes the strength of binding between two molecules. It has units of concentration and is inversely proportional to binding affinity.

Immunoassay: A method of molecular quantification that utilizes antibody-antigen interactions to achieve detection.

Limit of Detection (LOD): The lowest concentration that is statistically discernable from background signal.

Sensitivity: In this context, sensitivity refers to the ability of an assay to distinguish between small changes in concentration.

Specificity: In this context, specificity refers to the ability of an assay to discriminate between non-cognate and cognate molecules. 


\begin{abstract}
Analytical technologies based on binding assays have evolved substantially since their inception nearly 60 years ago, but our conceptual understanding of molecular recognition has not kept pace. Indeed, contemporary technologies such as single-molecule and digital measurements have challenged, or even rendered obsolete, core aspects of the conventional wisdom related to binding assay design. Here, we explore the fundamental principles underlying molecular recognition systems, which we consider in terms of signals generated through concentration-dependent shifts in equilibrium. We challenge certain orthodoxies related to binding-based detection assays, including the primary importance of a low $K_{\mathrm{D}}$ and the extent to which this parameter constrains dynamic range and limit of detection. Lastly, we identify key principles for designing binding assays optimally suited for a given detection application.
\end{abstract}




\section{Molecular Quantification via Concentration Dependent Shifts in Equilibrium}

The first published implementation of a binding-based molecular assay was an immunoassay for insulin detection in 1960 [1]. Over the following 60 years, analytical technologies based on binding assays have evolved substantially. Molecular detection platforms based on enzyme-linked immunosorbent assays (ELISAs) as well as newer commercial technologies, such as Luminex and NanoString, are core components of today's diagnostic armamentarium. In general, binding assays generate signal through concentration-dependent shifts in equilibrium that result from the interaction of the target molecule with an affinity reagent. Typically, either the target or affinity reagent is directly coupled to a moiety that generates an observable readout upon binding. For example, myriad immunosensors [2] have been developed that couple the interaction between antibody and antigen to an observable output based on an electronic [3], optical [4], or mechanical [5] signal, or even on the production and amplification of a quantifiable DNA sequence [6]-[8]. In all of these cases, an increase in target concentration shifts the equilibrium toward the formation of more antibody-antigen complexes, increasing the signal. Microarray technology likewise quantifies gene expression in a manner where the equilibrium fraction of target molecules bound to the array surface - and thus the fluorescence signal generated at their cognate array feature-is proportional to their concentration in the bulk solution [9]-[11].

A conventional understanding of molecular recognition principles has been used for decades to describe microarray and immunoassay technologies. However, the advent of contemporary molecular detection technologies, such as single-molecule measurements [12]-[16], has rendered important aspects of this understanding either unnecessarily restrictive or even obsolete. For instance, it is commonly assumed that the useful detection range of an affinity reagent spans an 81-fold change in target concentration centered around the equilibrium 
dissociation constant $\left(K_{\mathrm{D}}\right)$. This heuristic is still useful for conventional immunoassays, but it may be overly limiting in the broader context of molecular detection assays because it implies that one can only quantify target concentrations as low as $K_{\mathrm{D}} / 9$. There is also a prevalent notion that a lower $K_{\mathrm{D}}$ is always "better" because it results in a lower limit of detection (LOD), however there are many scenarios in which this would be detrimental to the assay. This is not to say that the conventional wisdom about molecular recognition is wrong, but rather that stringent adherence to prior conventions limits what could otherwise be achieved with newly available technologies. Here, we aim to reevaluate the conceptual frameworks used when developing binding assays and to provide an intuitive understanding of molecular recognition, empowering researchers to make better use of contemporary technologies for quantitative analysis of biological systems. A number of excellent articles have already shown how to shift and manipulate binding curves from a physicochemical perspective [17]. Therefore, we endeavor to approach the topic from a stance that is agnostic to the specific assay chemistry or implementation. After providing a brief background on the fundamental principles of affinity-based assays, we use simple mathematical reasoning and literature examples to argue against some long-held preconceptions of molecular recognition.

\section{Single-site molecular recognition}

Although more complicated examples of molecular recognition such as population shift [18]-[20], allostery [21]-[23], and proximity assays [24] have proven extremely powerful, many novel insights about assay development can still be gleaned from a simple model of bimolecular association. In the standard model of bimolecular association, the binding strength between an affinity reagent, $A$, and a target molecule, $T$, is characterized by the binding affinity, which is quantified in terms of $K_{\mathrm{D}}$ (eq. 1). 


$$
A+T \stackrel{K_{\mathrm{D}}=\frac{[A][T]}{[A \cdot T]}}{\Longleftarrow} A \cdot T
$$

Binding affinity plays a crucial role in determining what range of target concentrations yields an observable signal (Figure 1A). A typical antibody-antigen interaction has a $K_{\mathrm{D}}$ of $\sim 1 \mathrm{nM}[25]$. An affinity reagent is generally considered high affinity when $K_{\mathrm{D}}<10 \mathrm{nM}$ and low affinity when $K_{\mathrm{D}}$ $>1 \mu \mathrm{M}$, although, as we will discuss, this is not necessarily the best conceptual approach. The binding curve is commonly defined in terms of the Langmuir isotherm [26]-[28], an equation that characterizes the amount of binding as a function of target concentration (eq. 2). It is not without limitation (Box 1.). Several key assumptions underlie the simplicity of the Langmuir isotherm: i) single-site binding, ii) single $K_{\mathrm{D}}$, iii) one component is in excess, and iv) no off-target reactions. If these assumptions hold, one can derive the fraction of affinity reagent bound to target as

$$
f=\frac{[A \cdot T]}{[A]_{\text {total }}}=\frac{[T]}{K_{\mathrm{D}}+[T]}
$$

for conditions in which $[\mathrm{A}]<<[\mathrm{T}]$. This simple equation has informed much of our conventional understanding of molecular recognition, e.g. that $50 \%$ of affinity reagent molecules are bound to target when the target concentration is equal to the $K_{\mathrm{D}}$ (Figure 1B), which defines the center of the dynamic range around the affinity. In this context, the useful detection range has conventionally been defined as the range of target concentrations at which $10-90 \%$ of the affinity reagents are bound to target [17]. A cocktail-napkin analysis reveals that the detection range in this model spans target concentrations from $0.11-9.0 \times K_{\mathrm{D}}$, representing an 81-fold concentration window (Figure 1b). This implies that an affinity reagent with a $K_{\mathrm{D}}$ of $1 \mathrm{nM}$ can only 
quantitatively detect targets in the range of $111 \mathrm{pM}$ to $9 \mathrm{nM}$. However, the utility of this heuristic is called into question by the myriad examples of affinity reagents being used to quantify target concentrations far below their $K_{\mathrm{D}}$.

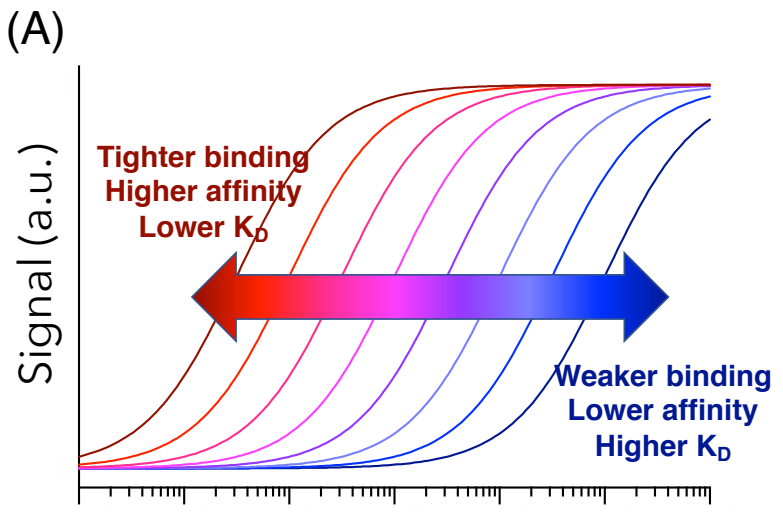

Target Concentration

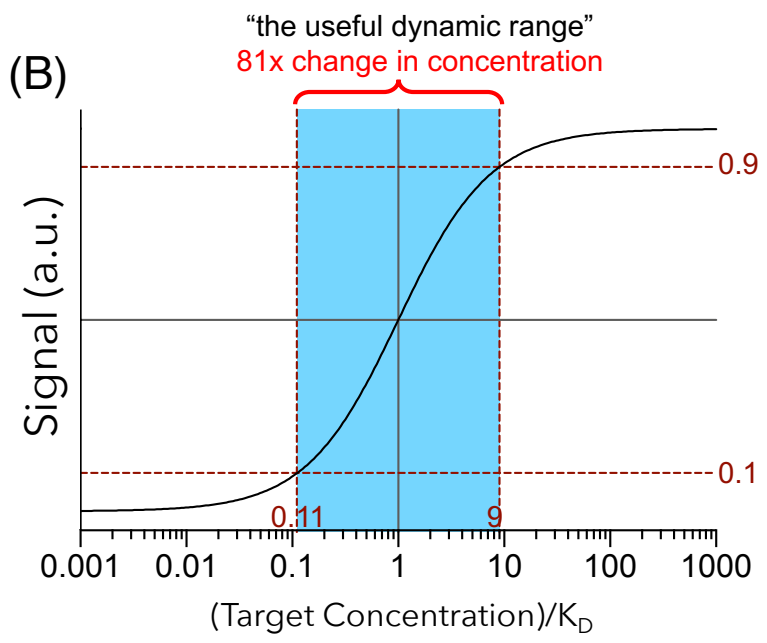

Figure 1 | Conventional understanding of binding curves. (a) Increasing affinity (i.e., lowering $K_{\mathrm{D}}$ ) shifts binding curves leftward relative to target concentration, whereas lowering affinity (i.e., increasing $K_{\mathrm{D}}$ ) leads to a rightward shift in the binding curve. (b) The "useful detection range" (blue) is considered to encompass the target concentrations over which $10-90 \%$ of the affinity reagent molecules are target-bound. This yields detectable target concentrations that span $0.11-$ $9.0 \times K_{\mathrm{D}}$. 


\section{Box 1. When can we use the Langmuir isotherm and when does it fall apart?}

A core - but often overlooked - assumption enabling the use of the Langmuir isotherm is that one component of the system must be in vast excess of the other so binding does not cause depletion of one of the species. If the target is much more abundant than the affinity reagent (i.e., $[T] \gg[A]$ ), then changes in the target concentration lead to changes in the fraction of affinity reagent that is bound to the target:

$$
[A \cdot T]=\frac{[T]_{\text {total }}}{[T]_{\text {total }}+K_{\mathrm{D}}}[A]_{\text {total }}
$$

In this case, the resultant signal is non-linear in terms of target concentration but linear in terms of affinity reagent concentration. In contrast, if the affinity reagent is present at a much higher concentration than the target (i.e., $[A] \gg[T]$ ), then the fraction of bound target remains constant while the total amount of target varies:

$$
[A \cdot T]=\frac{[A]_{\text {total }}}{[A]_{\text {total }}+K_{\mathrm{D}}}[T]_{\text {total }}
$$

Here, the signal is non-linear in terms of affinity reagent concentration and linear in terms of target concentration. Since it is typically easier to couple a detection modality (e.g., a fluorescent or bioluminescent tag) to an affinity reagent than to the target, the background signal scales with $[A]$ in most experimental setups. Therefore, the affinity reagent-limited scenario usually exhibits a more favorable signal-to-noise ratio than the target-limited scenario. However, a practical consideration of imposing the condition that $[T] \gg[A]$ is that in order to measure low target concentrations, affinity reagent concentration must be even lower. Thus, in the absence of signal amplification, the detection limit is constrained by the detection modality rather than the affinity reagent.

The conventional Langmuir isotherm no longer holds if affinity reagent and target concentrations are comparable. In this case, the amount of target-affinity reagent complexes is a non-linear function of the concentrations of both components[49]:

$$
[A \cdot T]=\frac{1}{2}\left([A]_{\text {total }}+K_{\mathrm{D}}+[T]_{\text {total }}-\sqrt{\left([A]_{\text {total }}+K_{\mathrm{D}}+[T]_{\text {total }}\right)^{2}-4[A]_{\text {total }}[T]_{\text {total }}}\right) .
$$

If $[A]=[T]=K_{\mathrm{D}}$, rather than observing the intuitive $50 \%$ binding, one would instead observe $\sim 38.2 \%$ binding. The concentrations must be raised to $[A]=[T]=2 K_{\mathrm{D}}$ to achieve $50 \%$ binding.

The Langmuir isotherm can be a powerful tool for rationalizing and analyzing binding data, especially because it conveniently distills a binding curve into a single parameter. However, the misuse of the equation can lead to misinterpretations and erroneous conclusions.

\section{What is a "good" $\mathrm{K}_{\mathrm{D}}$ ?}

Other misconceptions related to molecular recognition stem from our own implicit biases.

One such bias is the concept of an objectively "better" $K_{\mathrm{D}}$; we are biased towards thinking that a lower $K_{\mathrm{D}}$ is intrinsically superior because we predominantly work under conditions where the $K_{\mathrm{D}}$ is higher than the concentration of the target we are trying to detect. In practice, the concept of a 
"better" $K_{\mathrm{D}}$ is only relevant in the context of a desired detection range. Many researchers, however, fall into the trap of optimizing for lower and lower LODs - and by proxy, lower and lower $K_{\mathrm{D}}$ without regard for the biological scenario in which the assay is to be used.

We often overlook the fact that quantifying albumin concentrations in the $1 \mathrm{mM}$ range using an affinity reagent with $K_{\mathrm{D}}=1 \mu \mathrm{M}$ is as challenging as - or, as we will discuss later, possibly even more challenging than - quantifying insulin concentrations in the $1 \mathrm{nM}$ range using a reagent with the same affinity (Figure $\mathbf{2 A}$ ). If the $K_{\mathrm{D}}$ is too high, there will be too little signal over the desired detection range; if the $K_{\mathrm{D}}$ is too low, the signal will be saturated. In either case, one loses the ability to differentiate changes in target concentration via shifts in equilibrium. What matters is matching the detection range to the clinical scenario at hand. Therefore, a good effective $K_{\mathrm{D}}$ or dynamic range can only be defined in terms of a given biological context. For example, an affinity reagent with a $K_{\mathrm{D}}$ of $1 \mu \mathrm{M}$ is perfect for detecting ATP in blood, since its basal level is on the order of $20 \mathrm{~nm}$ to $1 \mu \mathrm{M}[29]$. In contrast, an affinity reagent with $K_{\mathrm{D}}=1 \mathrm{pM}$ towards ATP would be functionally useless because any reasonable deviation in ATP concentration would not result in an observable change in binding. Unfortunately, it remains common in the realm of biosensor development to report $K_{\mathrm{D}}$ without any mention of the clinically relevant detection range.
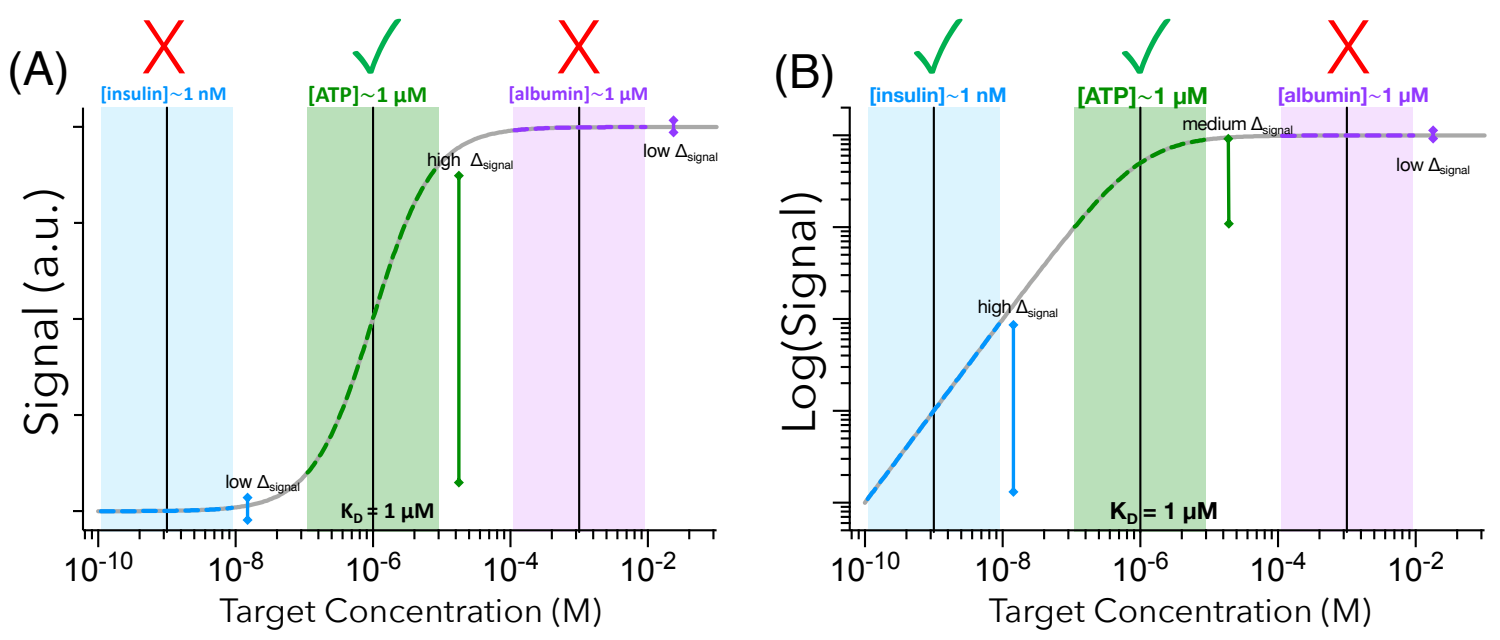
Figure $2 \mid$ The right $K_{\mathrm{D}}$ for the job. a) Detecting $1 \mathrm{nM}$ insulin with an affinity reagent with $K_{\mathrm{D}}=$ $1 \mu \mathrm{M}$ is just as challenging as detecting $1 \mathrm{mM}$ albumin. If the affinity is too high or too low, the output signal will not change appreciably with changes in target concentration. b) The greater sensitivity of contemporary single-molecule and digital detection assays means that one can, with sufficiently low background, quantitatively detect $1 \mathrm{nM}$ insulin more easily than $1 \mathrm{mM}$ albumin or even $1 \mu \mathrm{M}$ ATP using an affinity reagent with a $K_{\mathrm{D}}$ of $1 \mu \mathrm{M}$. In this scenario, a logarithmic scale for detection is more useful than the linear scale employed in $\mathbf{a}$.

\section{Logarithmic Detection Modalities and the Influence of Background Signal}

The advent of sophisticated detection modalities with extremely high resolution and considerably lower levels of background should encourage a reconsideration of how best to define signal output. In contrast to traditional readouts like bulk fluorescence or electrochemistry, which report ensemble averages of the number of bound affinity reagent molecules, single-molecule and digital assays report individual molecular counts. Since these outputs are quantitative over orders of magnitude, it is more useful to define the y-axis on a logarithmic scale. This has the important result that the quantification of analytes at concentrations arbitrarily lower than $K_{\mathrm{D}}$ becomes possible, assuming zero background signal. This is in stark contrast to the conventional thinking that the functional detection range is strictly proscribed relative to the $K_{\mathrm{D}}$. Moreover, the error associated with the calculated concentration decreases with the logarithm of the signal — that is, measurements get more accurate at lower concentrations (Figure 2B). Purely digital measurements with zero background are limited solely by Poisson statistics.

In the absence of background signal, the log dynamic range extends indefinitely, retaining quantitative resolution over arbitrarily low concentrations (Figure 3A). This means that in theory, the ability to detect low concentrations can be completely decoupled from $K_{\mathrm{D}}$. In practice, background signal strongly influences the LOD [30] and the detection range of an assay. Background signal can often be decreased through assay design or optimization whereas binding affinity is generally—with some exceptions[17], [18], [20], [23]—a fixed property of an affinity 
reagent-target pair. Moreover, a background signal of $1 \%$ has a large impact on a log detection range but a negligible effect on the conventional linear range (Figure 3B). Therefore, the minimization of background signal is more attractive for improving LOD than relying on decreasing $K_{\mathrm{D}}$. Specifically, assuming a constant coefficient of variation $(\mathrm{CV})$ for background signal, decreasing the background signal will have the same effect on the LOD as reducing $K_{\mathrm{D}}$ by the same factor (Figure 3C).
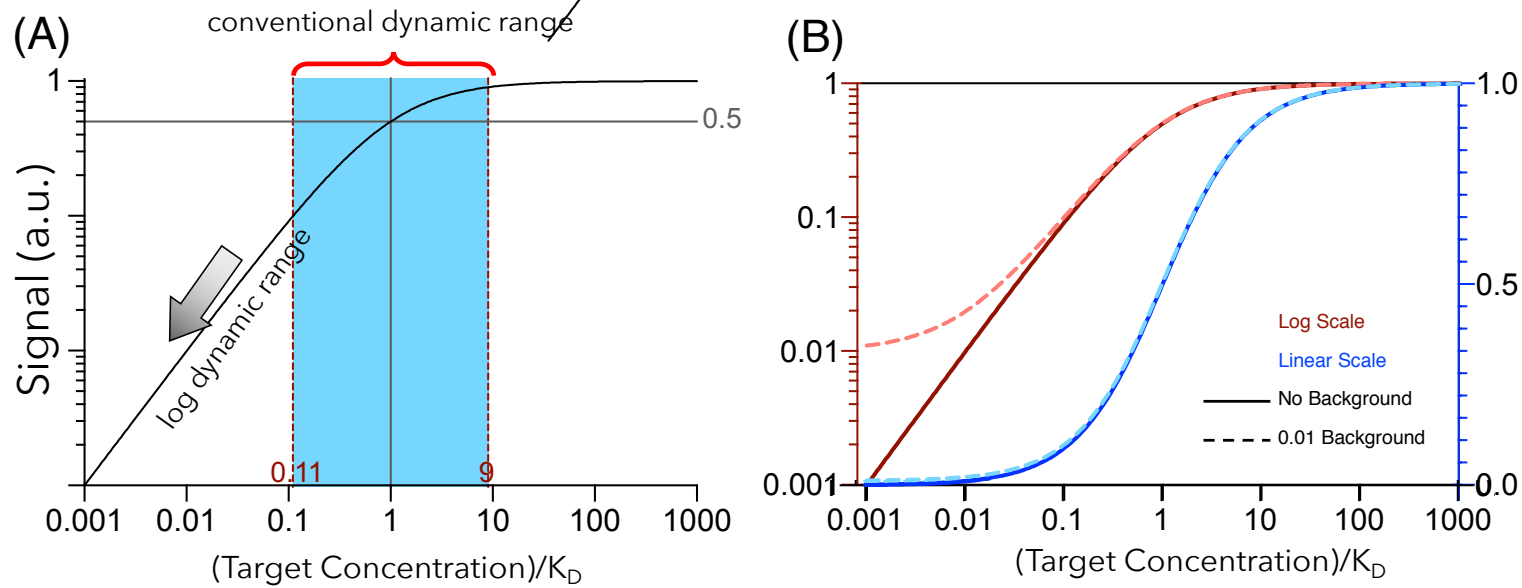

(C)

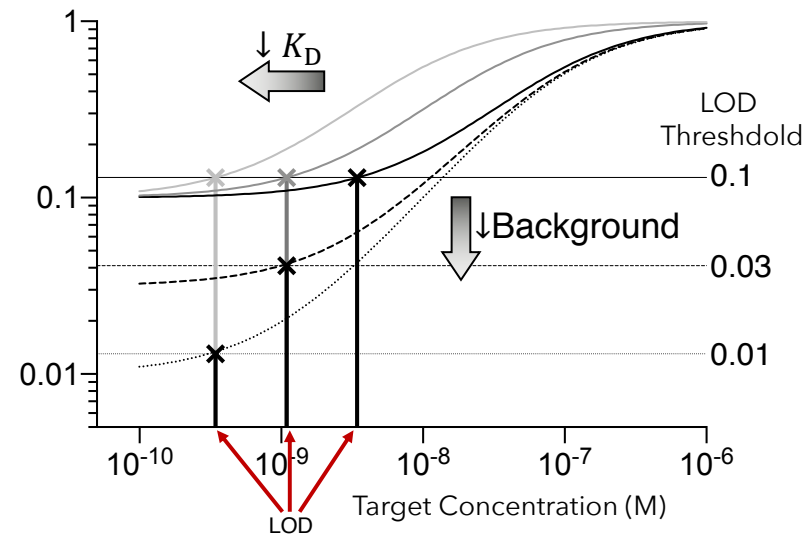

Figure 3 | Logarithmic detection and background signal. (A) If the y-axis can be plotted on a log-scale, which is appropriate for detection modalities that can distinguish between small changes in binding fraction, then the log-linear detection range extends below the conventional detection range (blue) as defined in Figure 1B. In the absence of background signal, the log detection range includes indefinitely low target concentrations and the center of the log dynamic range is no longer located at [Target] $=K_{\mathrm{D}}$. $(\mathbf{B})$ Background signal has a larger impact on a logarithmic output than a linear output. The extent to which the $\log$ detection range extends below $K_{\mathrm{D}}$ is a strong function of background signal. Adding a background signal of $1 \%$ does not appreciably affect the dynamic range for a linear output, but it greatly reduces the dynamic range for a log output. (C) Decreasing $K_{\mathrm{D}}$ (dark to light solid lines) has the same effect on LOD 
as decreasing the background signal by the same factor (solid to dashed to dotted black lines). Horizontal lines represent the background signals equivalent to 1,3 , and $10 \%$ plus three standard deviations, assuming $10 \%$ coefficient of variation. In contrast, $K_{\mathrm{D}}$ and background signal can have different effects on the detection range. Decreasing $K_{\mathrm{D}}$ does not appreciable change the extent of the detection range, whereas decreasing background signal greatly extends the log detection range.

Sophisticated detection modalities with considerably lower levels of background and digital outputs enable us to quantify molecules at concentrations vastly below $K_{\mathrm{D}}$, thereby rendering the traditional notion of an 81-fold detection range centered around the $K_{\mathrm{D}}$ obsolete. Instead, it is more useful to define a log-linear detection range where, given a sufficiently sensitive detection modality and low enough background, it should be possible to detect analytes at concentrations arbitrarily lower than the $K_{\mathrm{D}}$. A corollary of this rationale is that the burden of optimizing LOD should not be placed solely on decreasing the $K_{\mathrm{D}}$ of the affinity reagent itself but also on the development of low-background assays with detection modalities that can discriminate small changes (e.g., $0.1-0.01 \%)$ in the fraction of bound affinity reagents. Detection modalities that are capable of this level of resolution typically involve the counting of single-molecule events, such as droplet digital PCR [31], DNA sequencing [32], [33], digital ELISA [13], or singlemolecule imaging [15]. With a sufficiently low background signal and a logarithmic detection modality, it is possible to detect and quantify target concentrations many orders of magnitude below $K_{\mathrm{D}}$. An interesting ramification of this rationale is that the $K_{\mathrm{D}}$ of the affinity reagent must be higher than the physiological range of target concentration in order to ensure an appropriate log-linear detection range. Indeed, it is actually worse to have a $K_{\mathrm{D}}$ lower than the relevant concentration range.

\section{A Case Study}


This concept of extending the detection range through the selection of an appropriate detection modality and the elimination of background signal is exemplified by recent work from Li and colleagues [34], where they were able to consistently quantify analytes at concentrations that are orders of magnitude below $K_{\mathrm{D}}$. The authors used a three-state population-shift mechanism, which they termed recognition-enhanced metastably-shielded aptamer probes (RMSApt). The affinity reagents are designed in such a way that they can initiate a rolling circle amplification (RCA) reaction [35], [36] when bound to the target molecule. They are coupled to a solid surface, so that individual RCA products can be imaged and counted. This assay inherently exhibited high background signal, because aptamers not bound to target could also initiate an RCA reaction. The authors solved this problem by introducing an enzymatic "locking" mechanism that results in the non-equilibrium depletion of unbound aptamers, functionally eliminating background signal. Impressively, the authors observed quantitative detection ranges and LODs that consistently extend many orders of magnitude below the reported aptamer $K_{\mathrm{D}}$. These improvements in quantitative sensitivity appear to be very robust and were demonstrated with three different affinity reagents. An ochratoxin A (OTA) aptamer with a reported $K_{\mathrm{D}}$ of $500 \mathrm{nM}$ [37] exhibited a LOD of $38.8 \mathrm{fM}$ and a detection range of $100 \mathrm{fM}$ to $1 \mathrm{nM}$ (Figure 4A); a kanamycin aptamer with a reported $K_{\mathrm{D}}$ of $\sim 80 \mathrm{nM}$ [38] exhibited a LOD of $8.9 \mathrm{fM}$ and a detection range of $10 \mathrm{fM}$ to $10 \mathrm{pM}$ (Figure 4B); and a tyrosinamide aptamer with a reported $K_{\mathrm{D}}$ of $45 \mu \mathrm{M}$ [39] exhibited a LOD of $47.5 \mathrm{pM}$ and a detection range of $10 \mathrm{pM}$ to $100 \mathrm{nM}$ (Figure 4C). The authors consistently demonstrate the quantification of concentrations orders of magnitude below $K_{\mathrm{D}}$ ! 

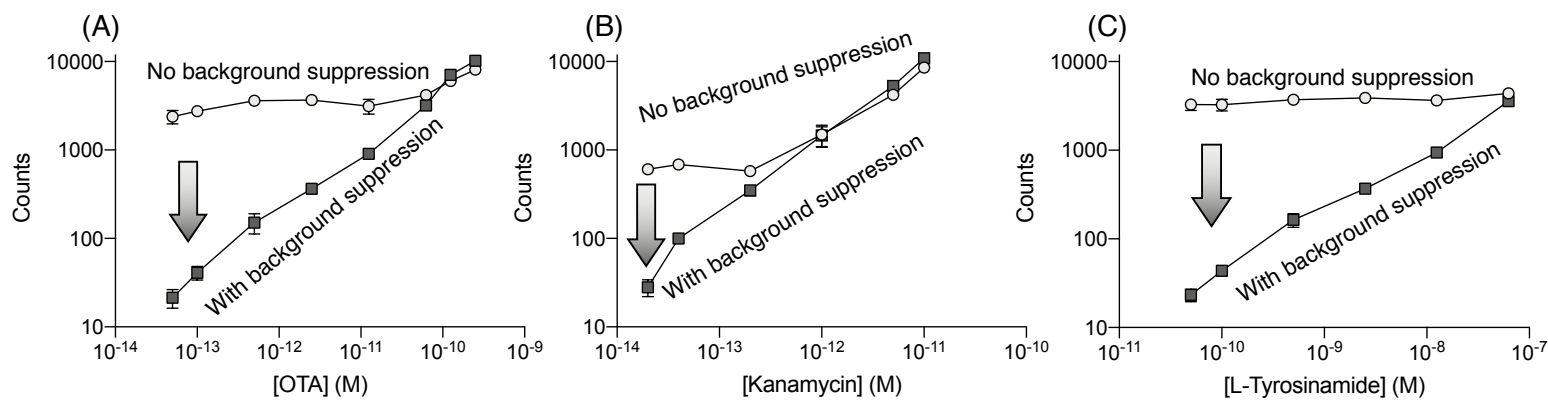

Figure $4 \mid$ Digital detection and low background single render the concept of an 81-fold detection range centered around the $K_{\mathrm{D}}$ obsolete. The recognition-enhanced metastably-shielded aptamer probe (RMSApt) system [34] exemplifies how a digital readout with little to no background signal on a log output scale can dramatically extend an assay's functional detection range. RMSApt essentially eliminates background signal through an enzymatic locking mechanism, achieving LODs and quantitative detection ranges that are orders of magnitude below reported affinities of the aptamers for their targets: a) ochratoxin A (OTA), b) kanamycin, and c) L-tyrosinamide. Importantly, a digital readout by itself (circles) is not sufficient to achieve these results; rather, a mechanism of background suppression (squares) must also be included. Data has been replotted from Ref. 36 with permission from the authors.

Although these results initially seem impossible in light of traditional understanding of molecular recognition, they should not be surprising considering the impact that background signal and the use of a digital detection modality have on the functional detection range. The high resolution is conferred in part by the ability to image and count individual binding events, which enables discrimination between small changes in the fraction of bound aptamers, and in part by the functional elimination of background through an enzymatic locking mechanism, which enables detection at concentrations far below $K_{\mathrm{D}}$. In this scenario, the ability to distinguish between small percentages of binding events is limited only by the number of viewable RCA products - which is in turn constrained by Poisson noise at the low end of target concentration and by image saturation at the high end. Thus, the ability to perform a high-sensitivity assay should not be exclusively dictated by access to high-affinity binding reagents but should also exploit a high-resolution detection modality and the ability to minimize background signal.

The previous example required the introduction of an irreversible reaction to the assay. This is not a necessity; many other methods can achieve similar outcomes. In another excellent 
example [40], the authors were able to detect and quantify fewer than 100 molecules/ $\mu \mathrm{L}$ simply by requiring that three antibodies bind to the target simultaneously in order to generate a signal. This equilibrium-based approach greatly reduced the probability of background signal generation and conferred ultra-high resolution. Other digital approaches based on molecular confinement [16], [41] have also illustrated the concepts discussed here, achieving successful quantitation at target concentrations far below the affinity of the binder.

\section{The Challenge of Specificity}

If the detection of analytes at concentrations orders of magnitude below the $K_{\mathrm{D}}$ is possible, is the effort [42], [43] to develop high affinity reagents unnecessary? This question seems to be particularly salient given the potential performance that can be achieved with a moderate-affinity binder and a focus on decreasing the background signal. However, there remains another important driving force providing the need for high-affinity detection reagents: specificity. Every biomolecule has an effective binding affinity to every other biomolecule, even though in most cases this affinity will be exceedingly low. Since most biomolecules can form multiple hydrogen bonds with one another, many off-target interactions will still produce a measurable $K_{\mathrm{D}}$ for a given affinity reagent, even if this affinity is many orders of magnitude weaker than that for the intended target. Since the concentrations of target molecules can be many orders of magnitude lower than the concentrations of off-target molecules [44], achieving high specificity through biomoleculemediated molecular recognition is critical. Even if one can still detect low target concentrations with modest affinity binders, the ability to distinguish between target and off-target molecules decreases as the affinity for on-target molecule decreases or as the concentration of off-target 
molecules increases. This becomes problematic if we do not understand the limitations imposed by imperfect specificity.

Therefore, we would like to conclude this perspective with a brief discussion on the limitations imposed by specificity. Imagine a scenario where an affinity reagent has a $K_{\mathrm{D} 1}$ of $1 \mu \mathrm{M}$ for a desired target, $T_{1}$. Assuming $\left[T_{1}\right] \gg[A]$, the log-linear signal is given by

$$
\log (f) \approx \log \left(T_{1}\right)-\log \left(K_{\mathrm{D} 1}\right)
$$

Upon the addition of an off-target molecule, $T_{2}$, for which the affinity reagent exhibits an affinity of $K_{\mathrm{D} 2}$, then the total signal is now approximately

$$
f_{\text {total }} \approx \frac{T_{1}}{K_{\mathrm{D} 1}}+\frac{T_{2}}{K_{\mathrm{D} 2}}
$$

assuming $\left[T_{2}\right] \gg[A],\left[T_{1}\right] \ll K_{D 1}$, and $\left[T_{2}\right] \ll K_{D 2}$. The log-linear range is now

$$
\log (f) \approx \log \left(T_{1}+\frac{K_{\mathrm{D} 1}}{K_{\mathrm{D} 2}} T_{2}\right)-\log \left(K_{\mathrm{D} 1}\right)
$$

In this scenario, measurements of $T_{1}$ in the presence of $T_{2}$ will be off by a factor of $\frac{K_{\mathrm{D} 1}}{K_{\mathrm{D} 2}} T_{2}$. This implies that the impact of off-target specificity becomes problematic if $T_{1} / T_{2}$ does not greatly exceed $K_{\mathrm{D} 1} / K_{\mathrm{D} 2}$. This reinforces the need for high affinity reagents. For example, consider the detection of a target that is present at $1 \mathrm{nM}$ in whole blood. Albumin is the off-target protein with the highest concentration in blood $(\sim 5 \mathrm{mM})$ [45]. If albumin binds weakly to the affinity reagent with $K_{\mathrm{D} 2} \sim 500 \mathrm{mM}$, then $K_{\mathrm{D} 1}$ would need to be less than $9 \mathrm{nM}$ for the error resulting from offtarget binding to albumin to be smaller than $10 \%$. As such, the concentrations and cross-reactivity of off-target molecules impose a lower limit on the affinity that is acceptable for an affinity reagent in a given context. This complication is often overlooked, perhaps because addressing it would require the impractical task of profiling each reagent's $K_{\mathrm{D}}$ for all possible off-target molecules. Perhaps confirmation bias is also at play; we have a tendency to seek confirming rather than 
disconfirming information [46]. This bias is exacerbated when unwarranted assumptions — such as the assumption that affinity reagents are highly specific — make disconfirming evidence seem unlikely. Fortunately, specificity can be increased through assay design. For example, by requiring multiple binding events to produce a signal [6], [40], [47], the probability of two binding events occurring on the same off-target molecule is greatly reduced. Therefore, specificity can be improved by requiring dual (or higher [40]) recognition for signal generation, an approach that has the added benefit of reducing background signal and therefore extending the log-linear dynamic range.

\section{Concluding Remarks}

A rigorous understanding of the core principles underlying binding assays is needed to guide the generation of affinity reagents that are best suited for the development of effective biosensors. Some of the conventional wisdom related to binding assays may be stifling the innovation that could be possible with novel technologies. For instance, we have discussed the limiting effects of over-reliance on $K_{\mathrm{D}}$ as a measure of biosensor performance and how our own biases cause us to overlook important factors like specificity because they are challenging to quantify. By considering binding assays in terms of concentration-dependent shifts in equilibrium, we have embarked on a critical assessment of the key features of these systems, with the goal of identifying important considerations for future biosensor development. First, we explored how the $K_{\mathrm{D}}$ of a given reagent for its target should only be judged as "better" or "worse" depending on the context in which it is to be employed. We showed that high-resolution measurements can be made even with a low affinity binder, and that by making use of a digital detector (in which binding events are counted rather than averaged in an ensemble) and a low-background assay design, one 
can extend the log-linear detection range to far lower target concentrations that are independent of $K_{\mathrm{D}}$ and limited only by Poisson noise. This is in stark contrast to the conventional view that an affinity reagent can only achieve quantitative detection at concentrations with a lower limit of $\sim K_{\mathrm{D}} / 9$. This implies that less effort should be spent developing high affinity binders and more on assay development. However, we also show that the upper limit of useful $K_{\mathrm{D}}{ }^{\prime} s$ for an affinity reagent is imposed by its specificity; higher concentrations of off-target molecules and stronger binding to these molecules necessitates the use of detection reagents with higher affinity for the target.

We conclude by noting that metrics such as $K_{\mathrm{D}}$, LOD, detection range, and specificity aren't the end of the story (see Outstanding Questions). Even with perfect molecular quantification, diagnostic assays are still limited by biological variability, which impacts clinical sensitivity and specificity [48]. Although the extent to which assay development should focus on LOD, sensitivity, specificity, or resolution will depend on the context in which the assay is to be used, the design of an optimal assay will inevitably entail achieving the right $K_{\mathrm{D}}$, a method of background suppression, a digital readout, and dual recognition (or some other mechanism to ensure specificity). It is our hope that the concepts presented here will instill an appreciation of the unexplored opportunities for pushing the diagnostic capabilities of our ever-increasing technological armamentarium.

\section{Acknowledgements}

We thank Dr. Evelin Sullivan of the Technical Communication Program at Stanford and Mr. Michael Eisenstein for their thoughtful comments and edits on the manuscript. 


\section{References}

[1] R. S. Yalow and S. A. Berson, "Immunoassay of endogenous plasma insulin in man," Obes. Res., vol. 4, no. 6, pp. 583-600, 1996.

[2] P. B. Luppa, L. J. Sokoll, and D. W. Chan, "Immunosensors - Principles and applications to clinical chemistry," Clin. Chim. Acta, vol. 314, no. 1-2, pp. 1-26, 2001.

[3] V. Mani, B. V. Chikkaveeraiah, V. Patel, J. S. Gutkind, and J. F. Rusling, "Ultrasensitive immunosensor for cancer biomarker proteins using gold nanoparticle film electrodes and multienzyme-particle amplification,” ACS Nano, vol. 3, no. 3, pp. 585-594, 2009.

[4] W. Lukosz, "Principles and sensitivities of integrated optical and surface plasmon sensors for direct affinity sensing and immunosensing," Biosens. Bioelectron., vol. 6, no. 3, pp. 215-225, 1991.

[5] D. Yang, A. Ward, K. Halvorsen, and W. P. Wong, "Multiplexed single-molecule force spectroscopy using a centrifuge," Nat. Commun., vol. 7, pp. 1-7, 2016.

[6] S. Fredriksson et al., "Protein detection using proximity-dependent DNA Ligation Assays," Nat. Biotechnol., vol. 20, no. May, pp. 473-477, 2014.

[7] P. Shahi, S. C. Kim, J. R. Haliburton, Z. J. Gartner, and A. R. Abate, “Abseq: Ultrahighthroughput single cell protein profiling with droplet microfluidic barcoding," Sci. Rep., vol. 7, no. March, p. 44447, 2017.

[8] T. Sano, C. L. Smith, and C. R. Cantor, "Immuno-PCR: Very sensitive antigen detection by means of specific antibody-DNA conjugates," Science (80-. )., vol. 258, no. 5079, pp. $120-122,1992$.

[9] C. Schröder et al., "Dual-color proteomic profiling of complex samples with a microarray of 810 cancer-related antibodies," Mol. Cell. Proteomics, vol. 9, no. 6, pp. 1271-1280, 
2010.

[10] C. Betzen, M. S. S. Alhamdani, S. Lueong, C. Schröder, A. Stang, and J. D. Hoheisel, "Clinical proteomics: Promises, challenges and limitations of affinity arrays," Proteomics - Clin. Appl., vol. 9, no. 3-4, pp. 342-347, 2015.

[11] R. Z. Gharaibeh, J. M. Newton, J. W. Weller, and C. J. Gibas, “Application of equilibrium models of solution hybridization to microarray design and analysis," PLoS One, vol. 5, no. $6,2010$.

[12] D. H. Wilson et al., "The Simoa HD-1 Analyzer," J. Lab. Autom., vol. 21, no. 4, pp. 533$547,2016$.

[13] L. Chang et al., "Single molecule enzyme-linked immunosorbent assays: Theoretical considerations," J. Immunol. Methods, vol. 378, no. 1-2, pp. 102-115, 2012.

[14] M. B. Scheible, G. Pardatscher, A. Kuzyk, and F. C. Simmel, "Single molecule characterization of DNA binding and strand displacement reactions on lithographic DNA origami microarrays," Nano Lett., vol. 14, no. 3, pp. 1627-1633, 2014.

[15] G. K. Geiss et al., "Direct multiplexed measurement of gene expression with color-coded probe pairs," Nat. Biotechnol., vol. 26, no. 3, pp. 317-325, 2008.

[16] D. M. Rissin et al., "Single-molecule enzyme-linked immunosorbent assay detects serum proteins at subfemtomolar concentrations," Nat. Biotechnol., vol. 28, no. 6, pp. 595-599, 2010.

[17] F. Ricci, A. Valle, A. J. Simon, A. Porchetta, and K. W. Plaxco, "Using Nature 's ' Tricks ' To Rationally Tune the Binding Properties of Biomolecular Receptors,” 2016.

[18] B. D. Wilson, A. A. Hariri, I. A. P. Thompson, M. Eisenstein, and H. T. Soh, "Independent control of the thermodynamic and kinetic properties of aptamer switches," 
Nat. Commun., vol. 10, no. 1, p. 5079, 2019.

[19] A. J. Simon, A. Vallée-Bélisle, F. Ricci, H. M. Watkins, and K. W. Plaxco, "Using the population-shift mechanism to rationally introduce 'hill-type' cooperativity into a normally non-cooperative receptor," Angew. Chemie - Int. Ed., vol. 53, no. 36, pp. 9471$9475,2014$.

[20] A. Vallee-Belisle and and K. W. P. Francesco Ricci, "Thermodynamic basis for the optimization of binding-induced biomolecular switches and structure-switching biosensors," Proc. Natl. Acad. Sci., vol. 106, no. 33, pp. 13802-13807, 2009.

[21] A. J. Simon, A. Vallée-Bélisle, F. Ricci, and K. W. Plaxco, "Intrinsic disorder as a generalizable strategy for the rational design of highly responsive, allosterically cooperative receptors," Proc. Natl. Acad. Sci. U. S. A., vol. 111, no. 42, pp. 15048-15053, 2014.

[22] J. D. Munzar, A. Ng, M. Corrado, and D. Juncker, "Complementary oligonucleotides regulate induced fit ligand binding in duplexed aptamers," Chem. Sci., vol. 8, no. 3, pp. 2251-2256, 2017.

[23] A. Vallée-Bélisle, F. Ricci, and K. W. Plaxco, "Engineering biosensors with extended, narrowed, or arbitrarily edited dynamic range," J. Am. Chem. Soc., vol. 134, no. 6, pp. 2876-2879, 2012.

[24] R. Y. Nong et al., "Solid-phase proximity ligation assays for individual or parallel protein analyses with readout via real-time PCR or sequencing," pp. 7-10, 2015.

[25] G. W. Litman and R. A. Good, Comprehensive Immunology (Volume 5, Immunoglobulins), 1st ed. PLENUM MEDICAL BOOK COMPANY, 1978.

[26] D. Colquhoun, "The quantitative analysis of drug-receptor interactions: A short history," 
Trends Pharmacol. Sci., vol. 27, no. 3 SPEC. ISS., pp. 149-157, 2006.

[27] A. V. Hill, "The mode of action of nicotine and curari determined by the form of the contraction curve and the method of temperature coefficients," J. Physiol., vol. 39, pp. 361-373, 1909.

[28] E. C. Hulme and M. A. Trevethick, "Ligand binding assays at equilibrium: Validation and interpretation," Br. J. Pharmacol., vol. 161, no. 6, pp. 1219-1237, 2010.

[29] M. W. Gorman, E. O. Feigl, and C. W. Buffington, "Human plasma ATP concentration," Clin. Chem., vol. 53, no. 2, pp. 318-325, 2007.

[30] G. L. Long and J. D. Winefordner, "Limit of Detection: A Closer Look at the IUPAC Definition," Anal. Chem., vol. 55, no. 7, pp. 712A-724A, 1983.

[31] L. Miotke, B. T. Lau, R. T. Rumma, and H. P. Ji, "High Sensitivity Detection and Quantitation of DNA Copy Number and Single Nucleotide Variants with Single Color Droplet Digital PCR,” 2014.

[32] T. Kivioja, A. Vähärautio, K. Karlsson, M. Bonke, S. Linnarsson, and J. Taipale, “Counting absolute number of molecules using unique molecular identifiers," Nat. Preced., 2011.

[33] K. Shiroguchi, T. Z. Jia, P. A. Sims, and X. S. Xie, "Digital RNA sequencing minimizes sequence-dependent bias and amplification noise with optimized single-molecule barcodes,” Proc. Natl. Acad. Sci. U. S. A., vol. 109, no. 4, pp. 1347-1352, 2012.

[34] R. Deng et al., "Recognition-Enhanced Metastably Shielded Aptamer for Digital Quantification of Small Molecules," Anal. Chem., vol. 90, no. 24, pp. 14347-14354, 2018.

[35] V. V Demidov, "Rolling-circle amplification in DNA diagnostics: the power of simplicity.," Expert Rev. Mol. Diagn., vol. 2, no. 6, pp. 542-548, 2002. 
[36] M. G. Mohsen and E. T. Kool, "The Discovery of Rolling Circle Amplification and Rolling Circle Transcription,” Acc. Chem. Res., vol. 49, no. 11, pp. 2540-2550, 2016.

[37] J. A. Cruz-Aguado and G. Penner, "Determination of ochratoxin A with a DNA aptamer," J. Agric. Food Chem., vol. 56, no. 22, pp. 10456-10461, 2008.

[38] K. M. Song et al., "Gold nanoparticle-based colorimetric detection of kanamycin using a DNA aptamer," Anal. Biochem., vol. 415, no. 2, pp. 175-181, 2011.

[39] E. Vianini, M. Palumbo, and B. Gatto, "In vitro selection of DNA aptamers that bind Ltyrosinamide," Bioorganic Med. Chem., vol. 9, no. 10, pp. 2543-2548, 2001.

[40] E. Schallmeiner et al., "Sensitive protein detection via triple-binder proximity ligation assays," Nat. Methods, vol. 4, no. 2, pp. 135-137, 2007.

[41] D. M. Rissin and D. R. Walt, "Digital concentration readout of single enzyme molecules using femtoliter arrays and poisson statistics," Nano Lett., vol. 6, no. 3, pp. 520-523, 2006.

[42] E. T. Boder, K. S. Midelfort, and K. D. Wittrup, "Directed evolution of antibody fragments with monovalent femtomolar antigen-binding affinity," Proc. Natl. Acad. Sci. U. S. A., vol. 97, no. 20, pp. 10701-10705, 2000.

[43] J. Wang et al., "Particle display: A quantitative screening method for generating highaffinity aptamers," Angew. Chemie - Int. Ed., vol. 53, pp. 4796-4801, 2014.

[44] J. R. Kettman, C. Coleclough, J. R. Frey, and I. Lefkovits, "Clonal proteomics: One genefamily of proteins," Proteomics, vol. 2, no. 6, pp. 624-631, 2002.

[45] F. Lee, "Direct Spectrophotometric of Albumin in Human Determination Serum," Clin. Chem., vol. 11, no. 4, pp. 478-487, 1965.

[46] R. H. Thaler, “Anomalies: The January Effect," J. Econ. Perspect., vol. 1, no. 1, pp. $197-$ 
201, 1987.

[47] A. P. Frei et al., "Highly multiplexed simultaneous detection of RNAs and proteins in single cells," Nat. Methods, vol. 13, no. 3, pp. 269-275, 2016.

[48] M. D. Borysiak, M. J. Thompson, and J. D. Posner, "Translating diagnostic assays from the laboratory to the clinic: Analytical and clinical metrics for device development and evaluation," Lab Chip, vol. 16, no. 8, pp. 1293-1313, 2016.

[49] T. D. Pollard, "A Guide to Simple and Informative Binding Assays," Mol. Biol. Cell, vol. 21, no. 23, pp. 4061-4067, 2010. 